\title{
Fuzzy modeling and design for a 3D Crane
}

\author{
P. Petrehuş, Zs. Lendek, P. Raica \\ Department of Automation, Technical University of Cluj Napoca, \\ Memorandumului 28, 400114, Cluj Napoca, Romania (e-mails: \\ \{paul.petrehus, zsofia.lendek, paula.raica\}@aut.utcluj.ro).
}

\begin{abstract}
Cranes are used to move heavy cargo. While they are in general controlled by a human operator, automated systems are able to obtain more precise control. In this paper, we design a Takagi-Sugeno (TS) fuzzy controller for the crane. For this, first a TS fuzzy model of the crane is developed, and a TS observer is used to estimate the unmeasurable states. The observer is tested in simulation and on a laboratory-scale 3D crane, while the controller is tested in simulation.
\end{abstract}

Keywords: 3D Crane, discretization, fuzzy modelling, observer, controller

\section{INTRODUCTION}

The $3 \mathrm{D}$ crane is a complex electromechanical system used to move heavy cargo. In general, an operator moves the crane in the desired position. The acceleration or deceleration of the crane may cause undesirable load swing. The load swing can cause human accidents, can damage the crane or the load. To control the swing, the operators need experience in crane maneuvering. Automated systems can help the operator control the load swing or can even replace the operator, (Inteco, 2008). They can also achieve precise control and increased load speed positioning (Chang and Chiang, 2009).

Various control methods have been used to reduce the load swing. For example in (Chang and Chiang, 2008) the swing control is realized using a fuzzy-logic projection controller designed based on cart position and swing angle. Sawodnya et al. (2002) consider the crane as a robot and combine feedforward, feedback control, and disturbance estimation to achieve the desired position and swing control. Chang and Chiang (2009) used a PID controller for rapid positioning and a fuzzy-logic control with deadzone compensation when the crane is close to the goal for precise positioning and moving the load smoothly. Antic et al. (2012) designed a fuzzy-logic controller to reduce the load swing during positioning of the crane. Pauluk (2002) developed a robust control method for the crane swing and an LQ controller is used for the reference trajectory errors compensation. Pauluk et al. (2001) solve the control problem using time-optimal control.

TS fuzzy models can be used to represent a large class of nonlinear systems. This type of models consist of local linear models which can represent the local input-output relations of a nonlinear system. In this paper we use a Takagi-Sugeno fuzzy representation (Takagi and Sugeno, 1985) of the crane model to design a discrete time observer and a discrete time controller that stabilizes the 3D crane. We have chosen the TS fuzzy model based approach because of its efficiency with complex nonlinear systems. To obtain the TS model we use sector nonlinearity approach (Ohtake et al., 2001). In the last decades, several methods have been developed to analyze the stability or to design observers or controllers for TS models (Tanaka and Wang, 2001). The analysis and design conditions are usually formulated as linear matrix inequality (LMI) problems, which can be solved using available methods.

This paper is organized as follows: Section II presents the nonlinear mathematical model of the crane. Section III presents the discrete time model and the equivalent TS fuzzy model. In Section IV the TS fuzzy observer is designed and simulation and experimental results for the designed observer are presented. Section V presents the TS fuzzy controller and the simulation results. Section VI provides the conclusions.

\section{3D CRANE}

The INTECO 3D Crane consists of a cart, moving on the $x y$ plane, and a payload attached to a rope, which can be shifted up and down. The system is schematically presented in Fig. 1 (Inteco, 2008). For this crane a 3D mathematical model representing the movement of the system on all three axes is considered. The system has

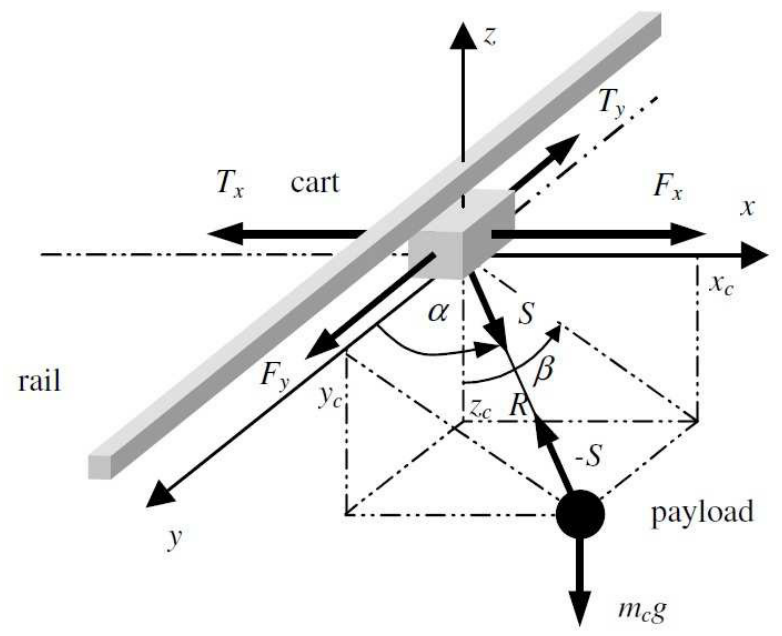

Fig. 1. 3DCrane system: coordinates and forces 
five measured quantities: $x_{w}$ denotes the distance of the rail of the cart from the center of the construction frame $[m], y_{w}$-distance of the cart from the center of the rail $[m]$ $\left(x_{w}\right.$ and $y_{w}$ are not represented in Fig. 1), $R$-length of the lift-line $[m], \alpha$-angle between the $y$ axis and the lift-line $[\mathrm{rad}], \beta$-angle between the negative direction on the $z$ axis and the projection of the lift-line onto the $x z$ plane $[\mathrm{rad}]$.

We adopt the following notations and model (Inteco, 2008):

$$
\begin{array}{ll}
x_{1}=y_{w} & x_{6}=\dot{x}_{5}=\dot{\alpha} \\
x_{2}=\dot{x}_{1}=\dot{y}_{w} & x_{7}=\beta \\
x_{3}=x_{w} & x_{8}=\dot{x}_{7}=\dot{\beta} \\
x_{4}=\dot{x}_{3}=\dot{x}_{w} & x_{9}=R \\
x_{5}=\alpha & x_{10}=\dot{x}_{9}=\dot{R} \\
T_{1}=\frac{T_{y}}{m_{w}}, T_{2}=\frac{T_{x}}{m_{w}+m_{c}}, T_{3}=\frac{T_{r}}{m_{c}} \\
u_{1}=\frac{F_{y}}{m_{w}}, u_{2}=\frac{F_{x}}{m_{w}+m_{c}}, u_{3}=\frac{F_{R}}{m_{c}} \\
N_{1}=u_{1}-T_{1}, N_{2}=u_{2}-T_{2}, N_{3}=u_{3}-T_{3} \\
\mu_{1}=\frac{m_{c}}{m_{w}}, \mu_{2}=\frac{m_{c}}{m_{w}+m_{s}}
\end{array}
$$

A simple nonlinear mathematical model of the crane with 3 control forces is:

$$
\begin{aligned}
& \dot{x}_{1}=x_{2} \\
& \dot{x}_{2}=N_{1}-\mu_{1} x_{5} N_{3} \\
& \dot{x}_{3}=x_{4} \\
& \dot{x}_{4}=N_{2}+\mu_{2} x_{7} N_{3} \\
& \dot{x}_{5}=x_{6} \\
& \dot{x}_{6}=\left(N_{1}-\mu_{1} x_{5} N_{3}-g x_{5}-2 x_{6} x_{10}\right) \frac{1}{x_{9}} \\
& \dot{x}_{7}=x_{8} \\
& \dot{x}_{8}=-\left(N_{2}+\mu_{2} x_{7} N_{3}+g x_{7}+2 x_{8} x_{10}\right) \frac{1}{x_{9}} \\
& \dot{x}_{9}=x_{10} \\
& \dot{x}_{10}=-N_{3}+g
\end{aligned}
$$

where $x_{1}, x_{2}, x_{3}, x_{4}, x_{5}, x_{6}, x_{7}, x_{8}, x_{9}, x_{10}$ are the system states. The system's variables and parameters are presented in Table I.

In order to have the equilibrium point in zero and to further simplify the mathematical model (1) we use the following change of variables:

$$
\begin{aligned}
& x_{9} \leftarrow x_{9}-0.1 \\
& u_{1}^{*} \leftarrow N_{1} \\
& u_{2}^{*} \leftarrow N_{2} \\
& u_{3}^{*} \leftarrow g-N_{3}
\end{aligned}
$$

We obtain:
Table 1. System variables and parameters

\begin{tabular}{|l|l|l|}
\hline Symbol & Meaning & Value \\
\hline \hline$u_{1}$ & Control in $y$ direction & - \\
\hline$u_{2}$ & Control in $x$ direction & - \\
\hline$u_{3}$ & Control along the lift-line & - \\
\hline$m_{c}$ & Mass of the payload $[\mathrm{kg}]$ & 1 \\
\hline$m_{w}$ & Mass of the cart $[\mathrm{kg}]$ & 2.49 \\
\hline$m_{s}$ & Mass of the moving rail $[\mathrm{kg}]$ & 4.09 \\
\hline$F_{x}$ & Force driving the rail with cart $[\mathrm{N}]$ & - \\
\hline$F_{y}$ & Force driving the cart along the rail $[\mathrm{N}]$ & - \\
\hline$F_{R}$ & Force controlling the length of the lift-line & - \\
& {$[N]$} & \\
\hline$T_{x}$ & Friction force $[N]$ & 100 \\
\hline$T_{y}$ & Friction force $[N]$ & 100 \\
\hline$T_{R}$ & Friction force $[N]$ & 75 \\
\hline$N_{1}$ & Resultant control in $x$ direction & - \\
\hline$N_{2}$ & Resultant control in $y$ direction & - \\
\hline$N_{3}$ & Resultant control in $z$ direction & - \\
\hline$g$ & Gravitational constant $\left[\mathrm{m} / \mathrm{s}^{2}\right]$ & 9.81 \\
\hline$x_{1}$ & Position on $y$ axis $[\mathrm{m}]$ & - \\
\hline$x_{2}$ & Velocity on $y$ axis $[\mathrm{m} / \mathrm{s}]$ & - \\
\hline$x_{3}$ & Position on $x$ axis $[\mathrm{m}]$ & - \\
\hline$x_{4}$ & Velocity on $x$ axis $[\mathrm{m} / \mathrm{s}]$ & - \\
\hline$x_{5}$ & Angle on $x$ axis $[\mathrm{rad}]$ & - \\
\hline$x_{6}$ & Angular velocity on $x$ axis $[\mathrm{rad} / \mathrm{s}]$ & - \\
\hline$x_{7}$ & Angle on $y$ axis $[\mathrm{rad}]$ & - \\
\hline$x_{8}$ & Angular velocity on $y$ axis $[\mathrm{rad} / \mathrm{s}]$ & - \\
\hline$x_{9}$ & Position on $z$ axis $[\mathrm{m}]$ & - \\
\hline$x_{10}$ & Velocity on $z$ axis $[\mathrm{m} / \mathrm{s}]$ & - \\
\hline & & \\
\hline
\end{tabular}

$$
\begin{aligned}
& \dot{x}_{1}=x_{2} \\
& \dot{x}_{2}=u_{1}^{*}+\mu_{1} x_{5} u_{3}^{*}-\mu_{1} g x_{5} \\
& \dot{x}_{3}=x_{4} \\
& \dot{x}_{4}=u_{2}^{*}-\mu_{2} x_{7} u_{3}^{*}+\mu_{2} g x_{7} \\
& \dot{x}_{5}=x_{6} \\
& \dot{x}_{6}=\left(u_{1}^{*}+\mu_{1} x_{5} u_{3}^{*}-\mu_{1} g x_{5}-g x_{5}-2 x_{6} x_{10}\right) \frac{1}{x_{9}+0.1} \\
& \dot{x}_{7}=x_{8} \\
& \dot{x}_{8}=-\left(u_{2}^{*}-\mu_{2} x_{7} u_{3}^{*}+\mu_{2} g x_{7}+g x_{7}+2 x_{8} x_{10}\right) \frac{1}{x_{9}+0.1} \\
& \dot{x}_{9}=x_{10} \\
& \dot{x}_{10}=u_{3}^{*}
\end{aligned}
$$

In Section III model (3) will be discretised and the equivalent TS fuzzy model is obtained.

\section{THE DISCRETE TIME FUZZY MODEL OF THE CRANE}

The 3D Crane system uses discrete time to measure the variables and consequently the continuous mathematical model (3) needs to be discretised. In this section we present the discrete time TS fuzzy system for the crane. We consider the parameter values presented in Table I.

\subsection{Model discretization}

We consider the notation: $q=\frac{1}{x_{9}+0.1}$.

System (3) can be written as

$$
\begin{aligned}
& \dot{\mathbf{x}}=A(\mathbf{x}) \mathbf{x}+B(\mathbf{x}) \mathbf{u} \\
& \mathbf{y}=C \mathbf{x}
\end{aligned}
$$


The system has five measured quantities, $y_{w}, x_{w}, \alpha, \beta$ and $R$ which are denoted in the mathematical model by $x_{1}, x_{3}, x_{5}, x_{7}$, and $x_{9}$. Therefore the output matrix is:

$$
C=\left(\begin{array}{llllllllll}
1 & 0 & 0 & 0 & 0 & 0 & 0 & 0 & 0 & 0 \\
0 & 0 & 1 & 0 & 0 & 0 & 0 & 0 & 0 & 0 \\
0 & 0 & 0 & 0 & 1 & 0 & 0 & 0 & 0 & 0 \\
0 & 0 & 0 & 0 & 0 & 0 & 1 & 0 & 0 & 0 \\
0 & 0 & 0 & 0 & 0 & 0 & 0 & 0 & 1 & 0
\end{array}\right)
$$

To obtain the discrete time system we use the Euler discretization:

$$
\begin{aligned}
A_{d} & =T_{e} A+I \\
B_{d} & =T_{e} B
\end{aligned}
$$

where $A$ and $B$ are the continuous system matrices, $I$ is the identity matrix having the same dimensions as $A$, $T_{e}$ represents the sampling time and $A_{d}$ and $B_{d}$ are the calculated discrete time matrices.

We chose the sampling time $T_{e}=0.01 \mathrm{~s}$, the same as the system data acquisition rate. Using (5) we obtain the discrete time system:

$$
\begin{aligned}
& \mathbf{x}(k+1)=A_{d}(\mathbf{x}(k)) \mathbf{x}(k)+B_{d}(\mathbf{x}(k)) \mathbf{u}(k) \\
& \mathbf{y}(k)=C \mathbf{x}(k)
\end{aligned}
$$

In the next section the equivalent TS fuzzy model of (6) is constructed using the sector non-linearity approach (Ohtake et al., 2001).

\subsection{Fuzzy model for the discrete time system (6)}

To obtain the TS model, we use the sector non-linearity approach (Ohtake et al., 2001). With this approach, a TS model equivalent to the original nonlinear model is built.

In (6), we have four nonlinear terms: $x_{5}(k), x_{7}(k), q(k)=$ $\frac{1}{x_{9}(k)+0.1}$ and $x_{10}(k)$ therefore the scheduling vector $z(k)=$ $\left(x_{5}(k), x_{7}(k), \frac{1}{x_{9}(k)+0.1}, x_{10}(k)\right)^{T}$. The system states are bounded, the bounds, based on the physical system are: $x_{5}(k) \in\left[-\frac{\pi}{2}, \frac{\pi}{2}\right], x_{10}(k) \in[-1,1], x_{7}(k) \in\left[-\frac{\pi}{2}, \frac{\pi}{2}\right]$ and $x_{9}(k) \in[0,0.9]$. The weighting functions for $z_{i}, i=1, \ldots, 4$ are constructed as follows:

(1) For $z_{1}(k)=x_{5}(k)$ the bounds are $z_{1, \min }=-\frac{\pi}{2}$ and $z_{1, \max }=\frac{\pi}{2}$. The weighting functions are $w_{11} \stackrel{2}{=}$ $\frac{z_{1, \max }-z_{1}}{z_{1, \max }-z_{1, \min }}$ and $w_{12}=1-w_{11}$. The term $z_{1}(k)$ can be written as $z_{1}(k)=z_{1, \min } w_{11}+z_{1, \max } w_{12}$.

(2) The term $z_{2}(k)=x_{7}(k)$ has the bounds $z_{2, \text { min }}=-\frac{\pi}{2}$ and $z_{2, \max }=\frac{\pi}{2}$. The weighting functions are $w_{21}=$ $\frac{z_{2, \max }-z_{2}}{z_{2, \max }-z_{2, \min }}$ and $w_{22}=1-w_{21} \cdot z_{2}(k)$ can be written as $z_{2}(k)=z_{2, \min } w_{21}+z_{2, \max } w_{22}$.

(3) $z_{3}=q(k)$ has the following bounds $z_{3, \min }=1$ and $z_{3, \max }=10$. The weighting functions are $w_{31}=$ $\frac{z_{3, \max }-x_{7}}{z_{3, \max }-z_{3, \min }}$ and $w_{32}=1-w_{31} \cdot z_{3}(k)=z_{3, \min } w_{31}+$ $z_{3, \max } w_{32}$.

(4) For $z_{4}(k)=x_{10}(k)$ the bounds are $z_{4, \min }=-1$ and $z_{4, \max }=1$. The weighting functions are $w_{41}=$ $\frac{z_{4, \max }-z_{4}}{z_{4, \max }-z_{4, \min }}$ and $w_{42}=1-w_{41} \cdot z_{4}(k)=z_{4, \min } w_{41}+$ $z_{4, \max } w_{42}$.

As we can see above, each scheduling variable $z_{i}, i=$ $1, \ldots, 4$ has 2 weighting functions, that means we have a fuzzy model with $2^{4}=16$ rules. The membership functions are computed as (Ohtake et al., 2001):

$$
h_{i}(z)=\prod_{j=1}^{p} w_{i_{j}}^{j}\left(z_{j}\right)
$$

with $i=1,2, \ldots, 2^{p}, i_{j} \in\{0,1\}, p$ is the number of nonlinearities, $r=2^{p}$ is the number of rules. The local linear models are obtained by replacing the correspondent values of the nonlinearities in matrices $A_{d}$ and $B_{d}$. For instance, one of the rules is:

Model rule 1: If $z_{1}(k)$ is $w_{11}$ and $z_{2}(k)$ is $w_{21}$ and $z_{3}(k)$ is $w_{31}$ and $z_{4}(k)$ is $w_{41}$ Then $\mathbf{x}(k+1)=A_{d_{1}} \mathbf{x}(k)+B_{d_{1}} \mathbf{u}(k)$

$$
A_{d_{1}}=\left(\begin{array}{ccccc}
1 & 0.01 & 0 & 0 & 0 \\
0 & 0.6988 & 0 & 0 & -0.0392 \\
0 & 0 & 1 & 0.01 & 0 \\
0 & 0 & 0 & 0.8860 & 0 \\
0 & 0 & 0 & 0 & 1 \\
0 & -0.3012 & 0 & 0 & -0.1373 \\
0 & 0 & 0 & 0 & 0 \\
0 & 0 & 0 & 0.1140 & 0 \\
0 & 0 & 0 & 0 & 0 \\
0 & 0 & 0 & 0 & 0
\end{array}\right.
$$

$$
\left.\begin{array}{ccccc}
0 & 0 & 0 & 0 & 0 \\
0 & 0 & 0 & 0 & 0 \\
0 & 0 & 0 & 0 & 0 \\
0 & 0.0149 & 0 & 0 & 0 \\
0.01 & 0 & 0 & 0 & 0 \\
1.02 & 0 & 0 & 0 & 0 \\
0 & 10.01 & 0 & 0 & \\
0 & -0.1130 & 1.02 & 0 & 0 \\
0 & 0 & 0 & 1 & 0.01 \\
0 & 0 & 0 & 0 & 1
\end{array}\right)
$$

$$
B_{d_{1}}=\left(\begin{array}{ccc}
0 & 0 & 0 \\
0.01 & 0 & 0.0063 \\
0 & 0 & 0 \\
0 & 0.01 & -0.0024 \\
0 & 0 & 0 \\
0.01 & 0 & 0.0063 \\
0 & 0 & 0 \\
0 & -0.01 & 0.0024 \\
0 & 0 & 0 \\
0 & 0 & -0.01
\end{array}\right)
$$

$\mathbf{x}(k+1)$ can be derived as:

$$
\mathbf{x}(k+1)=\sum_{j=1}^{16} h_{j}(\mathbf{z}(k))\left(A_{d_{j}} \mathbf{x}(k)+B_{d_{j}} \mathbf{u}(k)\right)
$$

$\mathbf{z}=\left[z_{i}\right]^{T}, i=1, \ldots, 4$.

The obtained TS system is the same as the original (6) in the considered limits.

\section{THE DISCRETE TIME FUZZY OBSERVER}

Applying a control law requires knowing the values of states. In practice it is not possible to measure all the system states. The solution for this problem are state observers. A state observer estimates the process states relying on the process mathematical model, using the input and the output of the process. 
In general, a Takagi-Sugeno fuzzy system has the form (Takagi and Sugeno, 1985):

$$
\begin{aligned}
& \mathbf{x}(k+1)=\sum_{i=1}^{r} h_{i}(\mathbf{z})\left(A_{i} \mathbf{x}(k)+B_{i} \mathbf{u}(k)\right) \\
& \mathbf{y}(k)=C \mathbf{x}(k)
\end{aligned}
$$

where $h_{i}$ are the membership functions, $A_{i}$-state matrix of rule $i, B_{i}$ are the input matrices, $C$ is the output matrix, $i=1 \ldots r, r$-number of rules, $\mathbf{x}=\left[x_{1}, x_{2}, \ldots, x_{n}\right]^{T}$ vector of systems states, $\mathbf{u}(k)=\left[u_{1}, u_{2}, \ldots, u_{m}\right]^{T}$-input, $\mathbf{y}=\left[y_{1}, y_{2}, \ldots, y_{l}\right]^{T}$-output of the system. Assuming that the scheduling variables are known, the general form of a fuzzy estimator is (Takagi and Sugeno, 1985):

$$
\begin{aligned}
& \hat{\mathbf{x}}(k+1)=\sum_{i=1}^{r} h_{i}(\mathbf{z}(k))\left(A_{i} \hat{\mathbf{x}}(k)+B_{i} \mathbf{u}(k)+\right. \\
& \left.+L_{i}(\mathbf{y}(k)-\hat{\mathbf{y}}(k))\right) \\
& \hat{\mathbf{y}}(k)=C \hat{\mathbf{x}}(k)
\end{aligned}
$$

where $L_{i}, i=1, \ldots, r$, are the observer gains. To design a stable estimator, we need to calculate positive definite matrices $P, H$ and $M_{i}, i=1 \ldots r$, solving the LMIs adapted from (Guerra and Vermeiren, 2004):

$$
\left(\begin{array}{cc}
-P & \left(H A_{i}-M_{i} C\right)^{T} \\
H A_{i}-M_{i} C & -H-H^{T}+P
\end{array}\right)<0
$$

$i=1 \ldots r, j=1 \ldots r$. The observer gains are calculated using the following relation:

$$
L_{i}=H^{-1} M_{i}, i=1, \ldots, r
$$

Solving (9) for system (6), we obtain 16 observer gains. For instance, the first gain is

$$
L_{1}=\left(\begin{array}{ccccc}
1 & 0 & -0.001 & 0 & 0 \\
0.08 & 0 & -0.08 & 0 & 0 \\
0 & 1 & 0 & 0.001 & 0 \\
0 & 0.02 & 0 & 0.1 & 0 \\
-0.02 & 0 & 1.2641 & 0 & 0 \\
-0.12 & 0 & 26.79 & 0 & 0 \\
0 & 0.002 & 0 & 1.26 & 0 \\
0 & 0.15 & 0 & 26.41 & 0 \\
0 & 0 & 0 & 0 & 1.06 \\
0 & 0 & 0 & 0 & 0.58
\end{array}\right)
$$

This observer by design guarantees that the estimation error converges asymptotically to zero. It has to be noted that for this application one of the scheduling variables is $x_{10}$, which is not measured, and therefore its estimated value has to be used in the observer. While the design of the observer does not take this situation into account, the designed observer is stable (i.e, the estimation error converges to zero) as long as the difference between the estimated and true initial states is small enough (Bergsten, 2001). This is confirmed both by simulation and experimental results.

First, we test the designed observer on simulated data. The initial conditions were $\mathbf{x}_{0}=[0.8381,0.0196,0.6813,0.3795$, $0.8318,0.7095,0.4289,0.3046,0.1897]^{T}$ and $\hat{\mathbf{x}}_{0}=0$. Fig. 2 presents the evolution of the system states for the inputs presented in Fig. 3. The evolution of error is presented in Fig. 4.

To test the observer on the physical system, the initial conditions were, for $\mathbf{x}_{0}=0$, the Home Point of the mechanical system, while the estimated initial states were $\hat{\mathbf{x}}_{0}=[0.1365,0.0118,0.8939,0.1991,0.2987$, $0.6614,0.2844,0.4692,0.0648,0.9883]^{T}$, a random point.

The measured data is presented in Fig. 6. To test the fuzzy observer on the crane we used a PID controller command as input. The estimates were calculated using the designed fuzzy estimator. The estimation error for the physical system is presented in Fig. 5.

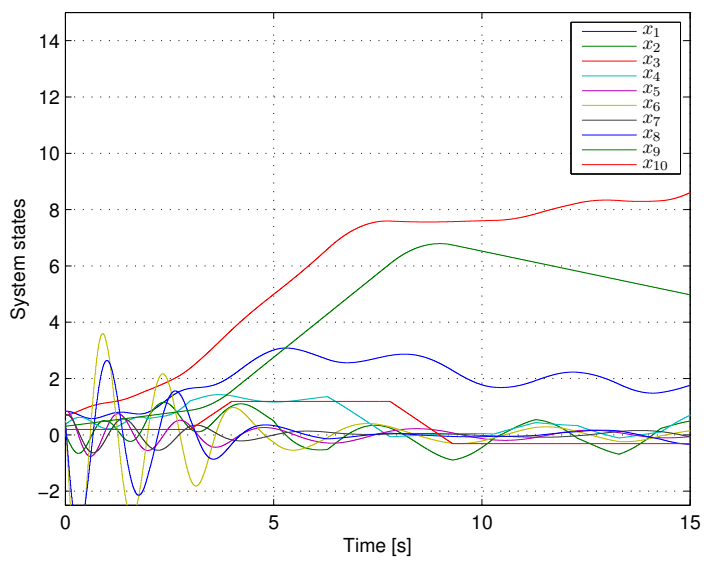

Fig. 2. Evolution of states (simulation)
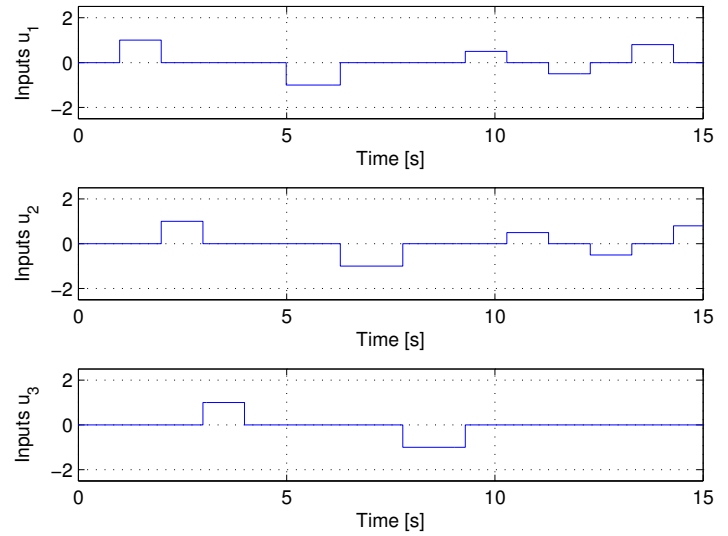

Fig. 3. System inputs (simulation)

Since the velocities are not measured, they are computed by differentiating the measured positions and filtering the result. In Fig. 7 we compare the filtered speed on $x$ direction using two filters, a zero-phase filter which can be used as a benchmark and a low-pass filter, and the estimated velocities. In this case we have a delay of 0.43 seconds between the low-pass filtered velocity and the estimated one. Between the zero-phase filtering on the $x$ velocity and the estimated velocity the differences are small. However, this filter can only be used offline.

The observer presented above has been designed without taking into account model uncertainties and measurement errors. Still the estimation errors are small enough for the observer to be suitable for this application.

In what follows, we design a TS controller for the 3D crane. 


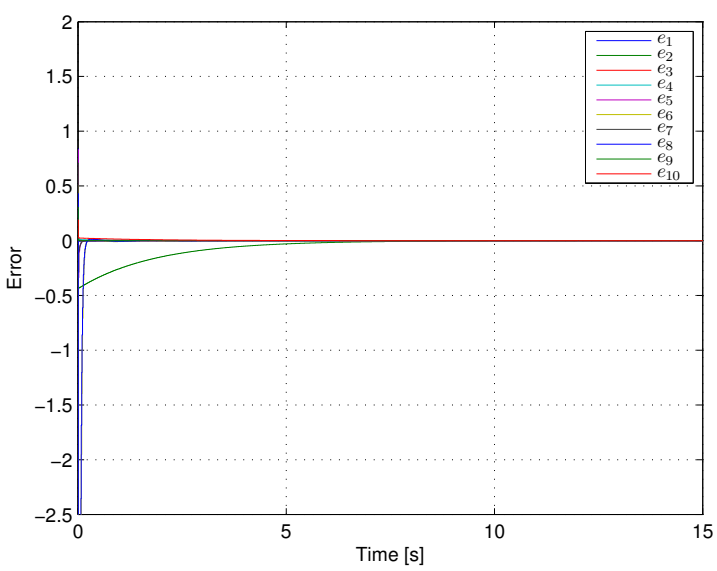

Fig. 4. Evolution of error (simulation)
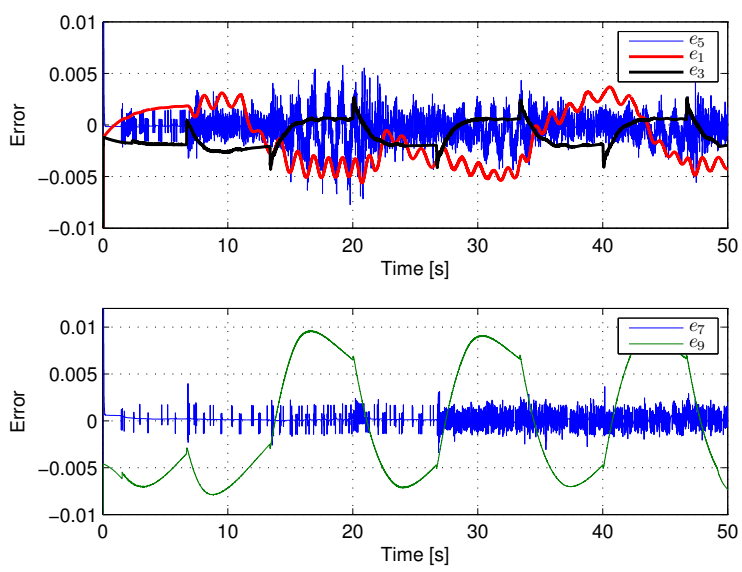

Fig. 5. Evolution of estimation error (experimental data)

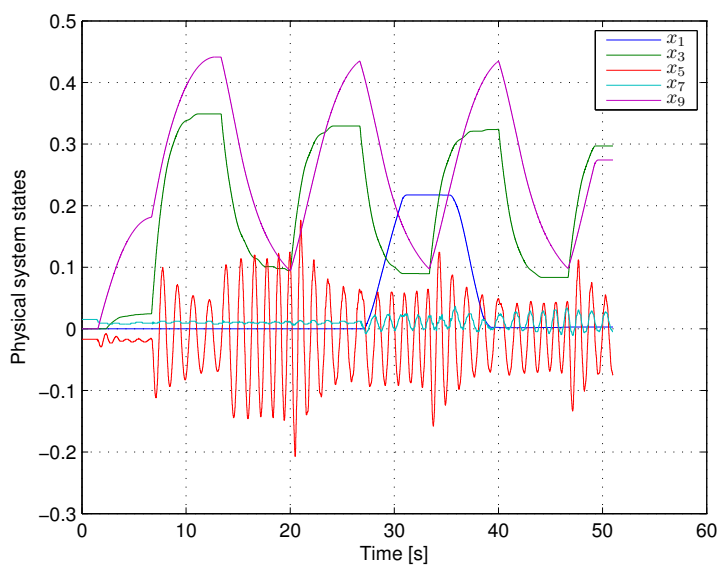

Fig. 6. Evolution of the physical system (experimental data)

\section{CONTROLLER DESIGN}

The discrete time fuzzy controller we use has the form:

$$
\mathbf{u}(k)=-\sum_{i=1}^{r} h_{i}(\mathbf{z}) F_{i} \mathbf{x}(k)
$$

where $F_{i}$ represent the local feedback gains, $i=1 \ldots r$.

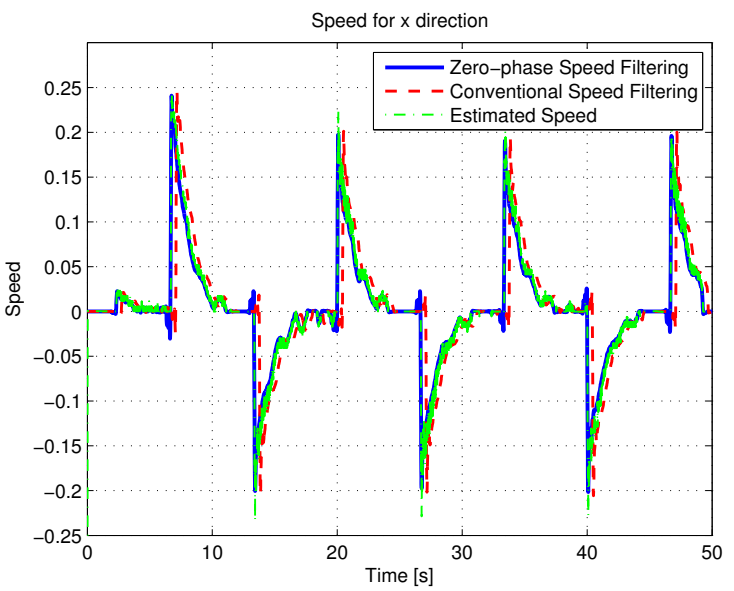

Fig. 7. Velocity on the $\mathrm{x}$ direction

Let

$$
G_{i j}=\left(\begin{array}{cc}
-H-H^{T}+P & \left(A_{i} H-B_{i} S_{j}\right)^{T} \\
A_{i} H-B_{i} S_{j} & -P
\end{array}\right)
$$

$i=1 \ldots r, j=1 \ldots r$. The closed loop system is stable if (Guerra et al., 2012):

$$
\begin{aligned}
& G_{i i}<0 \\
& \left(\frac{G_{i j}+G_{j i}}{2}\right)^{T}+\left(\frac{G_{i j}+G_{j i}}{2}\right)<0
\end{aligned}
$$

$i=1 \ldots r, j=1 \ldots r, i<j$.

The controller gains are calculated as:

$$
F_{i}=S_{i} H^{-1}, i=1, \ldots, r
$$

Solving the LMIs (11) for our system, we obtained 16 controller gains. For instance, the first gain is

$$
\begin{aligned}
& F_{1}=\left(\begin{array}{ccccc}
-2.72 & 24.44 & 0.08 & -0.04 & -18.92 \\
-0.17 & -0.69 & -2.49 & 5.92 & -3.05 \\
-0.27 & -0.95 & 0.31 & 0.49 & -5.15
\end{array}\right. \\
& \begin{array}{ccccc}
-7.52 & 2.21 & 0.43 & 0.1 & 0.14 \\
-0.18 & 20.48 & 7.47 & 0.02 & -0.01
\end{array} \\
& \left.\begin{array}{ccccc}
-0.18 & 20.48 & 7.47 & 0.02 & -0.01 \\
-0.53 & -2.34 & -0.21 & 2.31 & 4.51
\end{array}\right)
\end{aligned}
$$

This controller guarantees that the closed-loop system with all states known is asymptotically stabilized. If all system states can be measured then the developed controller can be connected to the process output and no observer is needed. To validate the controller we have tested this in simulation, and the evolution of the closed loop system is presented in Fig. 8. As can be seen, the system is stabilized.

Using the sensors on the electromechanical system we can only measure five quantities. Therefore we use the observer to estimate the unmeasured quantities, i.e. the velocities on the axes $\left(x_{2}, x_{4}, x_{10}\right)$ and the angular velocities $\left(x_{6}, x_{8}\right)$. The evolution of the closed loop system with the observer and controller is presented in Fig. 9.

In both situations the initial states were $\mathbf{x}_{0}=[0.2,0.2$, $0.04,0.1,0.1,0.18,0.14,0.11,0.12,0.02]^{T}$. The estimated initial states were $\hat{\mathbf{x}}_{0}=0$.

The time required to compute the estimated states and control input is $0.007 \mathrm{sec}$, which means that the observer based controller can be used online. 


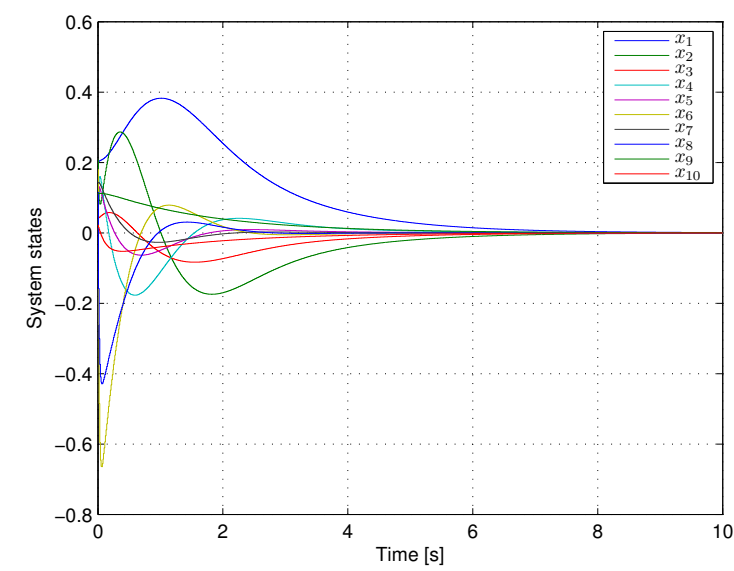

Fig. 8. Evolution of the closed loop control system (simulation)

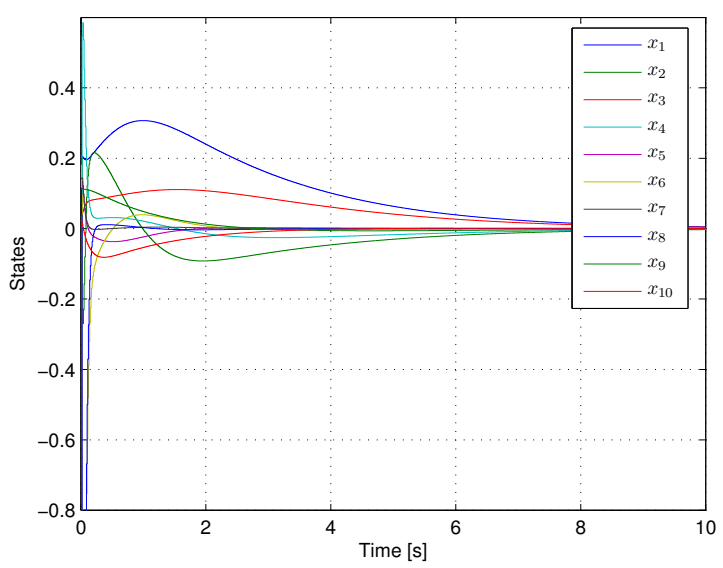

Fig. 9. Evolution the closed loop control system with observer (simulation)

\section{CONCLUSION}

In this paper we designed a discrete time fuzzy observer and controller for a 3D crane. The designed observer gives a good estimation of the system states and the estimation error is of a magnitude $10^{-3}$ on the physical system. In simulations, the controller stabilizes the system. In the future we will test the designed TS fuzzy controller on the physical system.

\section{ACKNOWLEDGEMENTS}

This work was supported by a grant of the Romanian National Authority for Scientific Research, CNCS - UEFISCDI, project number PN-II-RU-TE-2011-3-0043, contract number 74/05.10.2011.

\section{REFERENCES}

Antić, D., Jovanović, Z., Perić, S., Nikolić, S., Milojković, M., and Milošević, M. (2012). Anti-swing fuzzy controller applied in a 3D crane system. ETASR - Engineering, Technology \& Applied Science Research, 2, 196-200.
Bergsten, P. (2001). Observers and Controllers for TakagiSugeno Fuzzy Systems. Ph.D. thesis, Örebro University, Sweden.

Chang, C.Y. and Chiang, K.H. (2008). Fuzzy projection control law and its application to the overhead crane. Mechatronics, 18, 607-615.

Chang, C.Y. and Chiang, T.C. (2009). Overhead cranes fuzzy control design with deadzone compensation. Neural Comput \& Applic, 18, 749-757.

Guerra, T.M., Kerkeni, H., Lauber, J., and Vermeiren, L. (2012). An efficient Lyapunov function for discrete TS models: observer design. IEEE Transactions of Fuzzy Systems, 20(1), $187-192$.

Guerra, T.M. and Vermeiren, L. (2004). LMI-based relaxed nonquadratic stabilization conditions for nonlinear systems in the Takagi-Sugeno's form. Automatica, $40(5), 823-829$.

Inteco (2008). 3DCrane User's Manual. Available at www.inteco.com.pl.

Ohtake, H., Tanaka, K., and Wang, H. (2001). Fuzzy modeling via sector nonlinearity concept. In Proceedings of the Joint 9th IFSA World Congress and 20th NAFIPS International Conference, volume 1, 127-132. Vancouver, Canada.

Pauluk, M. (2002). Robust control of 3D Crane. Technical report, St. Staszic Technical University-AGH, Institute of Automatics.

Pauluk, M., Korytowsky, A., Turnau, A., and Szymkat, M. (2001). Time optimal control of 3D Crane. Technical report, St. Staszic Technical University-AGH, Institute of Automatics.

Sawodnya, O., Aschemannb, H., and Lahresc, S. (2002). An automated gantry crane as a large workspace robot. Control Engineering Practice, 10, 1323-1338.

Takagi, T. and Sugeno, M. (1985). Fuzzy identification of systems and its applications to modeling and control. IEEE Transactions on Systems, Man, and Cybernetics, 15(1), 116-132.

Tanaka, K. and Wang, H.O. (2001). Fuzzy Control System Design and Analysis: A Linear Matrix Inequality Approach. John Wiley \& Sons, New York, NY, USA. 